\title{
Quantum Invariance
}

\author{
Vasil Penchev, vasildinev@gmail.com \\ Bulgarian Academy of Sciences: Institute of Philosophy and Sociology: \\ Dept. of Logical systems and Models
}

\begin{abstract}
Quantum invariance designates the relation of any quantum coherent state to the corresponding statistical ensemble of measured results. The adequate generalization of 'measurement' is discussed to involve the discrepancy, due to the fundamental Planck constant, between any quantum coherent state and its statistical representation as a statistical ensemble after measurement.

A set-theory corollary is the curious invariance to the axiom of choice: Any coherent state excludes any well-ordering and thus excludes also the axiom of choice. It should be equated to a well-ordered set after measurement and thus requires the axiom of choice.

Quantum invariance underlies quantum information and reveals it as the relation of an unordered quantum "much" (i.e. a coherent state) and a well-ordered "many" of the measured results (i.e. a statistical ensemble). It opens up to a new horizon, in which all physical processes and phenomena can be interpreted as quantum computations realizing relevant operations and algorithms on quantum information. All phenomena of entanglement can be described in terms of the so defined quantum information.

Quantum invariance elucidates the link between general relativity and quantum mechanics and thus, the problem of quantum gravity.
\end{abstract}

Quantum mechanics offers an abundance of epistemological or ontological surprises seeming paradoxical at first glance, but contradicting the prejudices only. One of them, almost unknown, will be subject of this paper. It refers to the relation of ordering before and after measurement of any quantum system as well as the deducible conclusions and interpretations.

An initial way for quantum invariance to be defined is as follows: Any quantum coherent state before measurement cannot be a statistical ensemble excluding any "hidden variables" for the fundamental theorems of John von Neumann (1932) and of Simon Kochen and Ernst Specker (1967). Nevertheless the results after measurement constitute a statistical ensemble ordered in some finite values of a finite set of variables, e.g. the space-time coordinates of the measured results.

Consequently the following epistemological or ontological problems arise: How should map a coherent state (no statistical ensemble) before measurement with a statistical ensemble after measurement? Can just that mapping represent the original philosophical essence of quantum measurement? What are the definitive mathematical features of that mapping?

The answers have been long embarrassed by the following prejudices: The paradigm of measurement in classical physics supposes that the studied quantity has an exactly defined value before measurement, and the obtained statistical ensemble of the measured results is only due to various external disturbances or to the technical imperfections of the devices. The same model has been transferred implicitly and uncritically in quantum mechanics and measurements, and the following misleading philosophical interpretations have been made:

Quantum reality is incognizable or noncausal. It is created by or depends on its observer or measuring device and thus requires them. Quantum mechanics is incomplete and should complement by "hidden variables", yet unknown, to a future theory sharing cognizability, 
causality and independence from the observer and apparatus with the theories of classical physics and science. And so on. Their history is often called the philosophy of quantum mechanics. In fact all phenomena of entanglement studied by the theory of quantum information demonstrate bankruptcy of that philosophy of quantum mechanics since both "no hidden variables" and there are quantum correlations very well causally cognizable and independent from any observer or device.

The true pathway for the philosophy of quantum mechanics passes through the adequate generalization of 'measurement' to involve the discrepancy, due to the fundamental Planck constant, between any quantum coherent state and its statistical representation as a statistical ensemble by means of any measuring. That generalization neutralizing this discrepancy is meant as quantum invariance, which will be elucidated further.

The concept of measurement in classical physics may serve anyway as an initial reference frame, to which that generalization to be introduced. In fact the same problem of how the state before measurement to be mapped into the statistical ensemble of the measured results exists in classical physics and can be solved only for the above discrepancy is able to converge to zero: Indeed any statistical ensemble of results is finite and thus well-orderable while the corresponding physical quantity "before measurement" has to be smooth (and consequently continuous) so that its change to be described by differential equations. Only the axiom of choice is able to well-order it transforming it into the statistical ensemble of measured results. However the necessary collaboration of the axiom of choice is hidden since the difference between the real value before measurement and the corresponding element in the statistical ensemble of results converges to zero in principle for the Plank constant has no analog in classical physics.

That way out is closed to quantum mechanics: Any smooth quantity differs fundamentally from its measured value just for the Planck constant. However a usual "trick" in physics and mathematics is an arisen problem to be postulated as an axiomatic feature of the investigated system if it is consistent with the rest properties. So one has to require that any quantum coherent state is equivalent to the corresponding bounded, discrete, and consequently finite set of measured results, i.e. to a statistical ensemble, is an axiom in quantum mechanics.

A set-theory corollary is the curious invariance to the axiom of choice: Any coherent state excludes any well-ordering and thus excludes also the axiom of choice. However the above equivalence requires it to be equated to a well-ordered set after measurement and thus requires the axiom of choice for it to be able to be obtained. This is not a contradiction, though, since the statement (the axiom of choice) is in one situation (measurement), and its negation is in another (before measurement). Even the relation between the two situations does not generate contradictions, and it requires only the invariance in question.

In fact that invariance to the axiom of choice is well known in set theory as the so-called paradox of Skolem (1922). He introduces the relativity of 'set' meaning that any set of any power can be equivalently represented as a countable set utilizing the axiom of choice. Then if one considers a continuum in two axiomatic systems of set theory accordingly with or without the axiom of choice, the continuum would be invariant to the axiom of choice in the above sense. Furthermore Skolem states that the notions of finite or infinite set are thus relative (Skolem 1922 [1970]: [143-144]) and in the present context this already reaches a set-theory interpretation of quantum invariance. Indeed if the limit of any series is given, the axiom of choice guarantees that its element which differs from the limit by some finite value has to exist necessarily and thus a continuum to be effectively replaced by a discrete set.

In turn that invariance implies fundamental conclusions in the philosophy of quantum mechanics as it can be generalized as the specific relation between reality and cognition in 
quantum mechanics and designated as invariance to choice. The invariance to choice would resolve a series of philosophical problems in a way, in which they vanish as their alternatives turn out to be invariant to each other and thus redundant. However the generalization of the particular viewpoint of the philosophy of quantum mechanics beyond its domain seems rather problematic.

As to the proper area it implies radical conclusions, too: The physical sense and meaning of information, of its quantity as well as the smooth transition between physics and the physical, on the one hand, and mathematics and the mathematical, on the other, can be easily deduced:

The quantity of information turns out to be that one for the invariance of choice and this can be demonstrated well by the unit of information, a bit. For example, a bit can be represented as an empty cell (as on the tape of a Turing machine), in which two disjunctive options such as either " 0 " or " 1 " can be written and read. Then the invariance of that maximally elementary choice is one bit: The empty sell as an original "coherent state" and both certain results (either written or read) are equated and thus invariant as one bit of information. The bit can be generalized from two to infinitely many disjunctive options, which can be written or read on an empty sell, and designated as a quantum bit or qubit. If those options are represented as the written or read values of a unit sphere (i.e. on the surface of a unit ball), Hilbert space, which is the fundamental mathematical structure of quantum mechanics, is portrayed as that tape of a quantum Turing machine with infinitely many qubits instead of the finite number of bits of a standard Turing machine. Furthermore any wave function is just one state of that quantum Turing machine and thus any physical process being quantum in general turns out to be also an exactly defined quantum computation and vice versa.

The corresponding generalization of information is quantum information, which can be thought as that quantity apt to measure the qualitative quantum invariance. Then one qubit can be defined as that unit of quantum invariance, which is able to equate a coherent state to a countable set of measurable values for the state. Entanglement can be defined as that case when a coherent state is equivalent to any true subset of that countable set, i.e. entanglement decreases the degrees of freedom so that some measurable values of the coherent state are forbidden due to the external influence of one or more other coherent states.

All phenomena of entanglement studied by the theory of quantum information are describable as the relation of a qubit (or qubits) to its subset (their subsets). The relation can be transformed into the ratio between the corresponding measures of the subset and set since a qubit is a continuum of disjunctive options with nonzero measure as the area of the unit sphere.

So unlike the classically defined information, quantum information generates one derivative and additional metrics interpretable as the ratio between two qubits and thus as an angle between them and as an informational "curvature" available in all phenomena of entanglement.

The mathematical sense and meaning of that physically measurable ratio should be emphasized certainly: If the quantity of quantum information is visualized as a factor, which expresses a "much" as a "many" or "how many" per a unit of "much", that ratio being a finite number is the relation of an "infinitely many" to another "infinitely many" reducing the "common denominator" of one and the same "much". Thus quantum information allows of infinity to be represented physically and measured in an experimental way opening up to the horizon of an exact and quantitative science of infinity.

In particular gravity as it is mathematically represented in general relativity can be explained in terms of that ratio of qubits. These two qubits is enough to be accordingly the one of space-time position and the other of energy-momentum state in this position. Then their ratio will correspond to the space-time curvature in that point and to the value of gravitational field in it. 
Consequently gravity should have a quantum informational base and be relative to entanglement. Indeed entanglement and gravity can be seen correspondingly as the local and global aspect of one and the same, namely that variable metric relation embedded in quantum information.

That approach is consistent with the thermodynamic interpretation of general relativity (Jacobson1993) deducing it from the laws of classical thermodynamics and the Bekenstein bound (1973). In fact the latter introduces mutual incommensurability of the quantities, which are conjugates according to quantum mechanics like energy and time since their differentials are inversely proportional rather than proportional as in classical mechanics. So general relativity can be considered both as a quantum thermodynamic theory and as a theory of quantum gravity. However both the former and the latter are paradoxically realized:

General relativity as a thermodynamic theory has an inverse statistical representation in the following sense: If statistical thermodynamics reduces the whole of a thermodynamic system and its quantities to some statistical ensemble of elements like atoms or molecules and the quantities of their mechanical motions, general relativity is forced to reduce the elements of its ensemble whichever it is to the state or states of the system. The true space-time can be accepted as a statistical ensemble relevant to that general relativity interpreted thermodynamically. This reminds of some return of the "ether" removed by special relativity as redundant but consistent with general relativity (Einstein 1920).

So the approaches of statistical thermodynamics and general relativity turn out to be complement to each other or in other words, both together constitute a joint approach addressing a cyclic structure, in which the states of the system are identified as its parts and vice versa transferring an isomorphism between the Gibbs and Boltzmann interpretation of statistical mechanics.

General relativity as a theory of quantum gravity would quantize gravity in an extremely unexpected way: It is forced to consider any quantum whole including the universe effectively as a single quantum and representing its internality in a continuous or even smooth way. Said otherwise, general relativity offers an alternative and complementary viewpoint to describe any quantum leap and thus any mechanical motion in that continuous or even smooth way. Furthermore, that viewpoint can be quantitatively described as another law of conservation, which is the complementary counterpart of the fundamental conservation of energy (mass) in the framework of a more general conservation of action consistent with general relativity, in which energy-momentum is conserved only locally. If conservation of energy is "per a unit of time", its counterpart expresses the conservation of time (time period of a wave), which would be "per a unit of energy" describing how the total energy of the system is distributed between its parts. Both conservations can be interpreted as revealing a side of wave-particle duality and quantitatively complementing to each other in conservation of action. If the common conservation of energy (mass or energy-momentum) offers the usual perspective of particles localized in space-time, the newly conservation of time understands the world as a collection of coherent waves or of interacting states of the universe.

If the qubit is interpreted philosophically as "how many a much is", the relation of two qubits as a ratio is anyway problematic since this supposes a reduction of the "much" in two different qubits. Allowing that reduction, one can obtain an exactly defined value of curvature in any space-time point as above, but rejecting it the result is the common wave-particle duality of quantum mechanics expressing itself in incommensurable conjugates as well. General relativity determines any quantity relative to that ratio of qubits as a "bound variable" while quantum mechanics remains it as an empty degree of freedom or as a "free variable". General relativity and quantum mechanics are on both sides of another unifying fence derivative from that of 
quantum invariance: The former allows of two relations of quantum invariance to be commensurable by identifying their "much" as joint, the latter does not. One can say that both express differently one and the same: Globally the ratio of any two conjugates has to remain a free variable while any location of it determines an exactly defined value "bounding" the free variable and decreasing the degrees of freedom by one as if a "hologram" is projected on the "screen" of space-time.

The same ratio though introduced by general relativity can be interpreted in terms of quantum mechanics if wave-particle duality is temporally suspended as the ratio of the unit of smooth motion and that of quantum leap. Then quantum mechanics can be seen as that generalization of general relativity, which is apt to comprise also the discrete mechanical motions together with all smooth ones involved by the principle of relativity (Einstein 1918). However in that case the curvature of space-time could be interpreted as an equivalent smooth mapping of the variable ratio of those units. Consequently general relativity includes implicitly also all quantum leaps in another way. What it cannot involve in principle is wave-particle duality since this would be removed its subject, gravity.

Thus the concept of quantum invariance allows of the relation between those two, most fundamental physical theories to be seen in a new way and furthermore, the problem of quantum gravity as well: General relativity and the Standard model underlain by quantum mechanics are on both sides of quantum invariance, which unifies them so. All three interactions comprised by the Standard model constitutes a finite set of groups (i.e. symmetries) having representations in the group of the linear automorphisms of Hilbert space. Pseudo-Riemannian space utilized by general relativity to describe gravity can be obtained from Hilbert space by three reversible operations hinting the equivalence of both spaces under certain conditions: The first one transforms the actually infinitely dimensional Hilbert space into a process. The second replaces the independent variable of energy (frequency) with its reciprocal, time. The third one deforms both spheres and their relative position to each other corresponding to any given moment of time. If one omits to reverse the third operation, but does reverse the first and second one Hilbert space will be transformed into Banach space, and it will be able to represent entanglement as a mathematical formalism thus clearing up the close link between the structures associated with gravity and entanglement accordingly.

The concept of quantum invariance underlies that of quantum information and allows of revealing its philosophical and physical meaning as the relation of an unordered quantum "much" (i.e. a coherent state) and a well-ordered "many" of the measured results (i.e. a statistical ensemble). So quantum invariance clears up quantum information as a relation of ordering and opens up to a new horizon, in which all physical processes and phenomena can be interpreted as quantum computations realizing relevant operations and algorithms on quantum information. So one can peer into the "black box" of the physical world and discern its hidden mechanism, which turns out to be informational. Furthermore that concept shows up how quantum information is able to unify all known physical interactions situating gravity, on the one hand, and weak, strong and electromagnetic interaction, on the other hand, on both sides of quantum invariance. It needs entanglement to accomplish that unification and thus legitimates it as the bridge between these two sides and perhaps as a new fundamental interaction. Consequently the notion of quantum invariance has fundamental meaning for the philosophy of quantum mechanics and information. 


\section{References:}

Bekenstein, J. (1973). Black Holes and Entropy. Physical Review D, 7 (8), 2333-2346. doi 10.1103/PhysRevD.7.233.

Einstein, A. (1918). Prinziplelles zur allgemeinen Relativitätstheorie. Annalen der Physik, 55 (4), 241-244. doi:

Einstein, A. (1920). Äther und Relativitätstheorie. Berlin: Springer.

Jacobson, T. (1995). "Thermodynamics of Spacetime: The Einstein Equation of State," Physical Review Letters. 75 (7), 1260-1263. doi: 10.1103/PhysRevLett.75.1260.

Kochen, S. \& Specker, E. (1968). The problem of hidden variables in quantum mechanics. Journal of Mathematics and Mechanics. 17 (1), 59-87. doi: 10.1512/iumj1968.17.17004.

Neumann, J. (1932). Mathematische Grundlagen der Quantenmechanik. Berlin: Springer.

Skolem, T. (1922). Einige Bemerkungen zur axiomatischen Begründung der Mengenlehre. In E. Fenstad (ed.) Selected works in logic of Thoralf Skolem (pp. 137-152), Oslo: Univforlaget (1970). 